\title{
Correlation of CYP1A1, GSTP1 and GSTM1 gene polymorphisms and lung cancer risk among smokers
}

\author{
KYRIAKI PLIARCHOPOULOU ${ }^{1}$, GERASIMOS VOUTSINAS ${ }^{2}$, GEORGE PAPAXOINIS ${ }^{1}$, KATHERINE FLOROU $^{3}$, \\ MARIA SKONDRA ${ }^{1}$, KONSTANTINA KOSTAKI ${ }^{1}$, PARASKEVI ROUSSOU ${ }^{4}$, \\ KONSTANTINOS SYRIGOS ${ }^{4}$ and DIMITRIOS PECTASIDES ${ }^{1}$ \\ ${ }^{1}$ Oncology Unit, Second Department of Internal Medicine, Ippokrateion Hospital; \\ ${ }^{2}$ Laboratory of Environmental Mutagenesis and Carcinogenesis, Demokritos Biology Institute; \\ ${ }^{3}$ Laboratory of Molecular Biology, Ippokrateion Hospital; \\ ${ }^{4}$ Third Department of Internal Medicine, Sotiria Hospital, Athens, Greece
}

Received January 9, 2012; Accepted March 19, 2012

DOI: $10.3892 / \mathrm{ol} .2012 .665$

\begin{abstract}
Lung cancer is the leading cause of cancer mortality worldwide and tobacco smoking has been established as its biggest risk factor. Cigarette smoke contains several carcinogens. Most of them need to be activated by phase I enzymes, such as cytochrome P450 (CYP), while phase II enzymes, such as glutathione S-transferases are responsible for the detoxification of activated forms. The present study aimed to determine the role of CYP1A1, GSTP1 and GSTM1 gene polymorphisms in smoking-related lung cancer risk. It also aimed to investigate the association of the above polymorphisms with clinicopathological parameters, as well as their effect on survival. One hundred newly diagnosed lung cancer patients with advanced disease and 125 healthy controls with a smoking history participated in the study. The participants were screened for the presence of the following polymorphisms: MspI (CYP1A1), Ile105Val (GSTP1) and GSTM1 deletion. The above polymorphisms were also examined with regards to gender, age, histological type and survival. GSTP1 Ile/Val and GSTM1-null genotypes were associated with increased lung cancer risk and the presence of the combination of the three non-wild-type genotypes increases susceptibility to lung cancer (OR 3.328, 95\% $\mathrm{CI}=1.681-6.587, \mathrm{p}=0.001)$. In the non-small cell lung cancer group, the GSTP1 homozygous variant was significantly associated with increased lung cancer risk $(\mathrm{p}=0.008)$ and shorter survival. The results of this study suggest that the GSTP1 Ile/ Val genotype and GSTM1 deletion contribute to increased
\end{abstract}

Correspondence to: Dr Kyriaki Pliarchopoulou, Oncology Unit, Second Department of Internal Medicine, Ippokrateion General Hospital, Vasilissis Sofias 114, Athens 11527, Greece

E-mail:kpliarch@otenet.gr

Key words: lung cancer, polymorphisms, smoking, carcinogen metabolism lung cancer susceptibility. Moreover, GSTP1 Val/Val genotype is associated with increased lung cancer risk and shorter survival in non-small cell lung cancer patients.

\section{Introduction}

Lung cancer is the leading cause of cancer mortality accounting for 1.18 million deaths worldwide. The incidence rates have peaked among men and are continuing to rise among women, with half of the cases occurring in the developing world (1). Lung cancer is divided into two major types, nonsmall cell and small cell lung cancer. Small cell lung cancer accounts for $20 \%$ of all cases, while non-small cell lung cancer accounts for approximately $80 \%$ and includes three subtypes: Squamous-cell carcinoma, adenocarcinoma and large-cell undifferentiated carcinoma. Most of the patients are diagnosed at an incurable stage. Surgery is part of the management of the early stages of non-small cell lung cancer, while it plays a limited role in small cell lung cancer treatment. Patients with advanced disease usually only receive chemotherapy, with survival varying from a few weeks to a few years in non-small cell lung cancer and approximately one year for small cell lung cancer patients (2).

Tobacco smoking has been established as a major risk factor for lung cancer, although less than $20 \%$ of smokers are likely to develop the disease. The risk among smokers as compared with those who have never smoked is increased 8-15 times in men and 2-10 times in women and smoking cessation is known to reduce the risk, especially in heavy smokers (3). Cigarette smoke contains several chemicals which are known carcinogens, such as polycyclic aromatic hydrocarbons (PAHs), aromatic amines and N-nitroso compounds. Most of these chemicals are procarcinogens that need to be activated and converted into reactive carcinogens by phase I enzymes belonging to the cytochrome P450 gene superfamily (CYP). By contrast, phase II enzymes such as glutathione S-transferases (GSTs) are responsible for carcinogen detoxification. In the last two decades, medical and epidemiological studies have described genetic variants which appear to affect lung cancer 
susceptibility. Specifically, gene variants have been shown to be associated with increased lung cancer risk in smokers (4).

CYP1A1 is important in PAH and aromatic amine metabolism. The MspI polymorphism has been associated with increased lung cancer risk and is caused by one base substitution of thymine by cytosine in a non-coding region at position $3801\left(\mathrm{~T}^{3801} \mathrm{C}\right)$. Positive associations of CYP1A1 polymorphisms and lung cancer risk were identified in early Japanese studies (5). However, there is no consistent evidence of an association in other ethnic groups.

GSTM1 detoxifies PAH-derived epoxides by catalyzing their conjugation to glutathione. The GSTM1 null genotype causes loss of enzyme activity. GSTM1 deletion has been associated with host susceptibility in smoking-related lung cancer and the frequency of the deficiency ranges from 40-60\% in most ethnic groups (6).

Additionally, the GSTP1 genetic polymorphism is the result of a single base-pair substitution in which adenine is replaced by guanine leading to isoleucine replacement by valine in exon 5 (Ile ${ }^{105}$ Val). This substitution results in a disabled detoxification function and the accumulation of higher levels of activated carcinogens in the lung. Previous studies examining GSTP1 polymorphisms and lung cancer reported no significant association (7).

The primary objective of our study was to correlate CYP1A1, GSTM1 and GSTP1 polymorphisms and lung cancer risk in a Greek smoking population. Additionally, we examined the association of gene polymorphisms with clinical parameters and histology, as well as with survival.

\section{Patients and methods}

Patients. One hundred patients with histologically or cytologically documented advanced lung cancer (inoperable stage III and IV disease) of all histological types, and 125 healthy controls were included in the study. Informed consent was obtained from each participant. A detailed questionnaire was completed including past medical history, smoking and lifetime occupational history. Individuals with a history of previous environmental or occupational exposure were excluded. A medical check-up was carried out for the healthy individuals. Clinical characteristics were well-balanced between the two groups. The mean age was 67 and 68.5 years for the patient and control groups, respectively. The majority of the patients were male, representing $83 \%$ of the lung cancer patients and $86 \%$ of the healthy controls. The participants had a heavy smoking history of 62 and 58 packs/year for the two groups. Of the lung cancer cases, $52 \%$ had a diagnosis of adenocarcinoma, $24 \%$ of squamous cell carcinoma, $2 \%$ of large cell and $22 \%$ of small cell carcinoma (Table I). Additionally, all patients received platinum-based chemotherapy.

Blood samples and PCR. Blood samples were collected in EDTA-containing tubes from the participants and immediately stored at $-80^{\circ} \mathrm{C}$ until genotype analysis. Genomic DNA was isolated from the blood samples using standard procedures.

Following DNA isolation a PCR assay was used for each gene. The $\beta$-globin gene was co-amplified as a positive control for GSTM1 gene. The primers used were as follows: CYP1A1 forward: 5'-GATTGGGCACATGCTGACC-3',
Table I. Clinical characteristics of the participants.

Lung cancer patients Healthy controls

\begin{tabular}{lcc}
\hline Age (mean, years) & 67 & 68.5 \\
$\begin{array}{l}\text { Gender (m/f, \%) } \\
\begin{array}{l}\text { Smoking history } \\
\text { (packs/year) }\end{array}\end{array}$ & $83 / 17$ & $86 / 14$ \\
$\begin{array}{l}\text { Histology (\%) } \\
\text { Adenocarcinoma }\end{array}$ & 62 & \\
$\begin{array}{l}\text { Squamous cell } \\
\text { carcinoma }\end{array}$ & 24 & \\
$\begin{array}{l}\text { Large cell } \\
\text { carcinoma } \\
\text { Small cell }\end{array}$ & 2 & \\
carcinoma & 22 & \\
\hline
\end{tabular}

and reverse: 5'-CTGTCAAGGATGAGCCAGCA-3', GSTP1 forward: 5'-GTAGTTTGCCCAAGGTCGAG-3', and reverse: 5'-AGCCACCTGAGGGGTAAG-3', GSTM1 forward: 5'-GAA CTCCCTGAAAAGCTAAAGC-3', and reverse: 5'-GTTGG GCTCAAATATACGGTGG-3', $\beta$-globin forward: 5'-CAAC TTCATCCACGTTCACC-3', b-glob reverse: 5'-GAAGA GCCAAGGACAGGTAC-3'.

CYP1A1 gene PCR products were digested at $37^{\circ} \mathrm{C}$ overnight with MspI enzyme (Takara Bio, Inc., Shiga, Japan). Similarly GSTP1 gene PCR products were digested at $55^{\circ} \mathrm{C}$ for $2 \mathrm{~h}$ with $B s m a \mathrm{I}$ enzyme. The digestion products as well as GSTM1 PCR products were subjected to electrophoresis on a $2 \%$ agarose gel stained with ethidium bromide and the genotypes were determined by analysis of the bands on the gel. For CYP1A1 the presence of one band (340 bp) represented the homozygous wild type, three bands $(340,200$ and $140 \mathrm{bp})$ the heterozygous and two bands (200 and $140 \mathrm{bp}$ ) the homozygous variant. For GSTP1, the presense of one band (330 bp fragment) identified the homozygous for isoleucine (Ile/Ile), three bands (330, 220 and $110 \mathrm{bp}$ ) the heterozygous (Ile/Val) and two bands (220 and $110 \mathrm{bp}$ ) the homozygous for valine (Val/ Val). The absence of the band (210 bp) for GSTM1 indicated the GSTM1-null genotype, whereas the $\beta$-globin fragment (260 bp) confirmed the proper functioning of the reaction.

Statistical analysis. The association of the above polymorphisms with disease status (lung cancer versus healthy) was examined by the Chi-square or Fisher's exact test. Other associations were examined by the Chi-square or Fisher's exact test for categorical variables or the Mann-Whitney test for numerical variables. Patients were followed until they succumbed to the disease. Survival was defined as the time from the date of the initiation of chemotherapy until the date of death from any cause. Median survival was calculated using the Kaplan-Meier method. The prognostic significance of the above polymorphisms was examined using the log-rank test. Multivariate analysis of the prognostic factors was examined by the Cox-regression analysis. Variables were examined for their prognostic significance by multivariate analysis. All tests were two-tailed and a $p<0.05$ was considered statistically significant. 
Table II. Frequency of genotypes among lung cancer and healthy participants for CYP1A1, GSTP1 and GSTM1 genes.

\begin{tabular}{|c|c|c|c|}
\hline Gene status & Lung cancer patients & Healthy controls & p-value \\
\hline \multicolumn{4}{|l|}{ CYP1A1 } \\
\hline MspI (-/-) & 77 & 109 & 0.183 \\
\hline $\operatorname{MspI}(+/-)$ & 21 & 15 & \\
\hline $\operatorname{MspI}(+/+)$ & 2 & 1 & \\
\hline \multicolumn{4}{|l|}{ GSTP1 } \\
\hline Ile/Ile & 52 & 85 & 0.004 \\
\hline Ile/Val & 41 & 28 & \\
\hline $\mathrm{Val} / \mathrm{Val}$ & 7 & 12 & \\
\hline \multicolumn{4}{|l|}{ GSTM1 } \\
\hline Present & 45 & 79 & 0.011 \\
\hline Absent & 55 & 46 & \\
\hline
\end{tabular}

Table III. Survival and gene polymorphism status.

\begin{tabular}{lccc}
\hline Gene & Median survival (months) & 95\% CI (months) & \\
\hline CYP1A1 & & & \\
Wild-type & 15 & $12.2-17.8$ & \\
Heterozygous & 13 & $8.6-17.4$ & ns \\
Homozygous & 13 & & ns \\
GSTM1 & & $13.5-18.5$ & \\
Wild-type & 16 & $10.4-17.6$ & \\
Deficient & 14 & $14.5-21.5$ & ns \\
GSTP1 & & $10.9-17.1$ \\
Wild-type & 18 & $0.7-21.3$ \\
Heterozygous & 14 & & \\
Homozygous & 11 & & \\
\hline ns, not significant; CI, confidence interval. & & & \\
\hline
\end{tabular}

\section{Results}

Correlation of the genotype frequencies between the two groups. There was no statistically significant difference among the CYP1A1 wild-type, heterozygous and homozygous variants between the two groups. However, the heterozygous type for GSTP1 (Ile-Val) differed significantly, with $41 \%$ in the lung cancer group and $22 \%$ in the healthy controls $(p=0.004)$. Similarly, deletion of the GSM1 gene was shown in $55 \%$ of the lung cancer patients and $37 \%$ in the healthy control group $(\mathrm{p}=0.011)$ (Table II).

Results of the Cox regression analysis showed the presence of the heterozygous (Ile/Val) type [OR 2.46, 95\% confidence interval $(\mathrm{CI})=1.33-4.57, \mathrm{p}=0.004]$ and the GSTM1-null genotype (OR 2.081, 95\% $\mathrm{CI}=1.182-3.664, \mathrm{p}=0.011$ ) to be significantly associated with lung cancer. Additionally, the multivariate analysis revealed that the combination of wildtype genotypes for the three genes is correlated with reduced lung cancer risk by $69 \%(95 \% \mathrm{CI}=38-84 \%, \mathrm{p}=0.001)$, while the presence of non-wild type genotypes increases lung cancer susceptibility (OR 3.328, 95\% CI=1.681-6.587, $\mathrm{p}=0.001$ ).

Regarding the clinicopathological characteristics, the analysis demonstrated that there was no correlation between gene polymorphisms and gender, age or histological subtype. However, in the group of non-small cell lung cancer patients the only polymorphism significantly associated with increased lung cancer risk was the GSTP1 homozygous variant (HR $3.558,95 \% \mathrm{CI}=1.398-9.053, \mathrm{p}=0.008)$. There was no association between gene polymorphisms and lung cancer susceptibility in the small cell lung cancer group.

Correlation between survival and gene polymorphisms. In total, 98 patients were followed between 1 and 60 months until they succumbed to the disease, with two patients being lost to follow-up. Median survival was 15 months (95\% CI=12.917.1 months) (Table III).

As shown, there was no statistically significant correlation between genotypes and survival, with the exception of the 
homozygous variant of GSTP1 which was associated with a shorter median survival of 11 months $(\mathrm{p}=0.004)$. Similarly, in the non-small cell lung cancer group, patients carrying two copies of the valine allele for the GSTP1 gene had a significantly shorter median survival of 7 months, compared with 18 months for the wild-type genotype $(\mathrm{p}=0.001)$ and 14 months for the heterozygous type $(\mathrm{p}=0.043)$.

\section{Discussion}

Experimental research has demonstrated that PAHs and other carcinogens in smoke form DNA adducts that lead to the mutation of targets, such as the tumor-suppressor gene p53, and ultimately cause cancer (8). Epidemiological studies have also shown a good correlation between PAH- and aromatic-DNA adducts in blood and lung tissue of smokers (9). Additionally, there are reports of elevated concentrations of DNA adducts in white blood cells or lung tissue in cancer cases compared with controls (10). These data suggest that carcinogen activation may be a risk factor for cancer, while the detoxification of these carcinogens may play a protective role against DNA damage. Therefore, certain individuals would be more susceptible to DNA damage due to metabolic activation or decreased detoxification of environmental carcinogens (11).

CYP1A1 MspI polymorphism has been shown to increase catalytic activity for pro-carcinogen conversion, including PAH and aromatic amines. GSTP1 and GSTM1 catalyze glutathione conjugation with carcinogens leading to detoxification. There are a number of studies on gene polymorphisms with regards to lung cancer risk. However, the results of such studies have been inconsistent and some are contradictory (12-16). Therefore, in the present study, we examined the presence of the polymorphisms MspI for CYP1A1 gene, Ile105Val for GSTP1 and deletion for GSTM1 gene and their association with lung cancer incidence in Greek smokers.

Findings of this study showed that the frequency of the CYP1A1 genotype did not differ significantly between lung cancer patients and healthy controls $(p=0.183)$, while there was a statistically significant difference in the frequency of GSTP1 Ile/Val $(p=0.004)$ and GSTM1-deletion $(p=0.011)$ between the two groups.

In Caucasian populations, the $M s p \mathrm{I}$ polymorphism variants range from 15 to $33 \%$ with the $M s p I$ homozygous variant found to be between 0 and $4 \%$ (17). In our study, 22 and $12 \%$ were heterozygous and 2 and $1 \%$ were homozygous for MspI, in the lung cancer and control groups, respectively. Moreover, the homozygous deletion for GSTM1 is found to be approximately $50 \%$ in Caucasians (18). In the population of our study the percentage of GSTM1 deletion was 55\% for the lung cancer patients and $37 \%$ for the controls. Moreover, as shown in pooled analyses, in Caucasians, the wild-type genotype (Ile/ Ile) for GSTP1 is $47 \%$, the heterozygous-type (Ile/Val) is $42 \%$ and the homozygous mutant allele ( Val/Val) is approximately $11 \%$ (19). In our population the homozygous mutant allele was found to be 7 and $10 \%$ in the lung cancer cases and healthy controls, while the heterozygous type (Ile/Val) was 41 and $22 \%$, respectively. The difference in GSTP1 Ile/Val frequency between the two groups was statistically significant $(p=0.004)$.

The Cox regression analysis revealed that GSTM1 deletion as well as the heterozygous (Ile/Val) type for GSTP1 gene were associated with increased lung cancer risk. Conversely, no association was found for the $M s p \mathrm{I}$ polymorphism.

Our findings on the GSTM1 deletion are in agreement with previous studies. Several meta-analyses and pooled analyses have been consistent in finding a statistically significant increase in risk for individuals carrying the GSTM1 null genotype. A recent meta-analysis involving 19638 cases and 25266 controls from 98 studies revealed increased lung cancer risk (OR 1.22, 95\% CI=1.14-1.30), particularly, in individuals of an East Asian ethnic background (OR 1.38, 95\% CI=1.24-1.55) (20).

Given that GSTP1 is the most abundant isoform in the lungs, it is expected to be of particular importance in the detoxification of inhaled carcinogens. Individuals with the Ile/Val or Val/Val genotype exhibit significantly lower 'detoxification' activity compared with those with the Ile/Ile genotype. Additionally, carriers of at least one copy of the $G$ (guanine) allele have increased levels of hydrophobic adducts in the lung and higher levels of PAH-DNA adducts in human lymphocytes (21). A meta-analysis performed for all published papers revealed no association between lung cancer risk and the GSTP1 exon 5 polymorphism. However, the reports on the GSTP1 polymorphism do not demonstrate an increased lung cancer risk. By contrast, a pooled analysis of selected published studies suggested an increased risk of lung cancer associated with carrying at least 1 valine allele (OR 1.11, 95\% $\mathrm{CI}=1.03-1.21$ ). In addition, the GSTP1 exon 5 polymorphism varied according to ethnicity. When stratified by ethnicity, this association was strongly observed among Asians, especially, in those with adenocarcinoma, but not among Caucasian subjects (19).

A global meta-analysis of 71 case-control studies involving 30368 subjects was performed to evaluate the effect of CYP1A1 gene polymorphisms on lung cancer susceptibility. MspI polymorphism of the CYP1A1 gene was found to be a risk factor associated with increased susceptibility for lung cancer, but these associations vary between ethnic populations. The odds ratio of the MspI polymorphism for lung cancer was 1.19 (95\% CI=1.11-1.28). In the subgroup analysis by ethnicity, increased risk was observed among East Asians and only marginal associations were detected among Caucasians and other populations (22).

Several reports have evaluated the effects of combinations of risk genotypes on cancer susceptibility. In particular, GSTM1 deletion is a moderately strong susceptibility factor for cancer but may become a dominant risk factor in the presence of gene-gene combinations. Studies have clearly shown the dependence of adduct levels on the CYP1A1 genotype which is more pronounced in GSTM1-deficient smokers. Smokers with CYP1A1 MspI variant/GSTM1-null genotypes were found to have increased levels of DNA adducts in lung tissue and leucocytes (17). Furthermore, in a Greek study by Dialyna et al the combination of rare variants of the CYP1A1, GSTM1 and GSTT1 genes was over-represented in lung cancer patients, compared to the control population and was significantly associated with a history of heavy tobacco consumption in lung cancer patients (23). In our study, consistent with previous studies, the combination of the $M s p I$ variant and GSTM1 deletion was significantly associated with lung cancer risk $(p=0.029)$. Furthermore, our study demonstrated that the combination of the risk genotypes for the three genes 
significantly increases lung cancer susceptibility (OR 3.328, 95\% CI=1.681-6.587, $\mathrm{p}=0.001$ ).

Evidence regarding the correlation of polymorphisms with histological types is lacking. A study by Risch et al showed that the risk alleles for GSTM1 and GSTP1 were present at a higher frequency in squamous cell carcinoma than in adenocarcinoma. This appears to be consistent with the reduced detoxification of carcinogens in cigarette smoke which has been shown to be more important in the development of squamous cell lung cancer (24). Our study found no association between histological subtypes and the risk genotypes. However, when the genotypes were analysed according to the two main categories, non-small and small cell lung cancer, the homozygous (Val/Val) type for the GSTP1 gene was significantly associated with lung cancer risk in the non-small cell lung cancer group.

The literature on gene polymorphisms and survival is inconsistent $(25,26)$. In a study by Sweeney et al involving 274 lung cancer patients the GSTM1-null genotype was associated with shorter survival with a relative risk of death, adjusted for stage and histology, of 1.36 (95\% CI=1.04-1.80) (27). These results were consistent with those of another study by Goto et al who examined 232 non-small lung cancer patients and found a statistically significant worse survival in the group of patients with the GSTM1 deletion (28). In the same study, no effect on survival was observed by the GSTP1 genotype. In a previous study comprising 138 non-small cell lung cancer patients, the GSTP1 exon 5 and GSTM1 deletion polymorphisms were not associated with the clinical outcome (29). The lung cancer patients involved in our study had advanced-stage disease and had all received platinum-based chemotherapy. Following a 60-month follow-up, survival data were analysed. The gene polymorphisms were not correlated with clinical outcome, with the exception of GSTP1 Val/Val genotype which was associated with a shorter survival of 11 months $(p=0.004)$. The same genotype was also found to have a negative impact on survival in the non-small cell lung cancer group, compared with the wild-type and heterozygous genotypes.

Our study therefore demonstrated a significant impact of GSTP1 Ile/Val and Val/Val genotype on lung cancer susceptibility in a Caucasian population and associated the GSTP1 Val/ Val variant with survival deterioration.

In conclusion, the role of gene polymorphisms in the development of lung cancer has been extensively studied in several ethnic groups. We investigated the role of certain gene polymorphisms in the development of lung cancer in Greek individuals exposed to cigarette smoke. Additionally, we demonstrated a statistically significant lung cancer risk in patients with either a GSTM1 deletion or GSTP1 Ile/Val genotype. The homozygous variant for GSTP1 (Val/Val) was shown to be associated with increased risk among non-small cell lung cancer patients. Furthermore, the Val/Val genotype was correlated with a shorter survival $(\mathrm{p}=0.004)$ in the overall population, as well as in the non-small cell lung cancer group.

\section{References}

1. Parkin DM, Bray F, Ferlay J and Pisani P: Global cancer statistics, 2002. CA Cancer J Clin 55: 74-108, 2005.

2. Tamura T: New state of the art in small-cell lung cancer. Oncology (Williston Park) 15: 8-10, 2001.
3. Khuder SA and Mutgi AB: Effect of smoking cessation on major histologic types of lung cancer. Chest 120: 1577-1583, 2001.

4. Kiyohara C, Shirakawa T and Hopkin JM: Genetic polymorphism of enzymes involved in xenobiotic metabolism and the risk of lung cancer. Env Health Prev Med 7: 47-59, 2002.

5. Kawajiri K, Nakachi K, Imai K, Watanabe J and Hayashi S: The CYP1A1 gene and cancer susceptibility. Crit Rev Oncol Hematol 14: 77-87, 1993.

6. Benhamou S, Lee WJ, Alexandrie AK, Boffetta P, Bouchardy C, Butkiewicz D, Brockmöller J, Clapper ML, Daly A, Dolzan V, et al: Meta- and pooled analyses of the effects of glutathione S-transferase M1 polymorphisms and smoking in lung cancer risk. Carcinogenesis 23: 1343-1350, 2002.

7. Ye Z, Song H, Higgins JP, Pharoah P and Danesh J : Five glutathione S-transferase gene variants in 23452 cases of lung cancer and 30397 controls: meta-analysis of 130 studies. PLoS Med 3: 91, 2003.

8. Denissenko M, Pao A, Tang MS and Pfeifer GP: Preferential formation of benzo(a)pyrene adducts at lung cancer mutational hotspots in p53. Science 274: 430-432, 1996.

9. Wiencke J, Varkonyi A, Semey K, et al: Detection of benzene-related DNA adducts in human tissues and evidence of co-activation with polyaromatic hydrocarbons. Proc Am Assoc Cancer Res 36: 135, 1995.

10. Kriek E, Van Shooten FJ, Hillebrand MJX, Van Leeuwen FE, Den Engelse L, De Looff AJ and Dijkmans AP: DNA adducts as a measure of lung cancer risk in human exposed to polycyclic aromatic hydrocarbons. Env Health Perspect 99: 71-75, 1993.

11. Bouchardy C, Benhamou S, Jourenkova N, Dayer P and Hirvonen A: Metabolic genetic polymorphisms and susceptibility to lung cancer. Lung Cancer 32: 109-112, 2001.

12. Raunio H, Husgafvel-Pursianinen K, Anttila S, Hietanen E, Hirvonen A and Pelkonen O: Diagnosis of polymorphisms in carcinogen-activating and inactivating enzymes and cancer susceptibility-a review. Gene 159: 113-121, 1995.

13. Perera FP, Mooney LA, Stampfer M, Phillips DH, Bell DA, Rundle A, Cho S, Tsai WY, Ma J, Blackwood A and Tang D: Associations between carcinogen-DNA damage, glutathione S-transferase genotypes and risk of lung cancer in the prospective Physicians' Health Cohort Study. Carcinogenesis 23: 1641-1646, 2002.

14. Kumar M, Agarwal SK and Goel SK: Lung cancer risk in north Indian population: role of genetic polymorphisms and smoking. Mol Cell Biochem 322: 73-79, 2009.

15. San Jose C, Cabanillas A, Benitez J, Carrillo JA, Jimenez M and Gervasini G: CYP1A1 gene polymorphisms increase lung cancer risk in a high-incidence region of Spain: a case-control study. BMC Cancer 10: 463, 2010.

16. Sampaio AC, Morari EC, Bufalo NE, Leite JL, Lima CS and Ward LS: Lack of influence of glutathione S-transferase genotype profile on cancer susceptibility in smokers and non-smokers. Med Sci Monit 15: 10-15, 2009.

17. Bartsch H, Nair U, Risch A, Rojas M, Wikman H and Alexandrov K: Genetic polymorphism of CYP genes, alone or in combination, as a risk modifier of tobacco-related cancers. Cancer Epidem Biom Prev 9: 3-28, 2000.

18. Hung RJ, Boffetta P, Brockmoller J, Butkiewicz D, Cascorbi I, Clapper ML, Garte S, Haugen A, Hirvonen A, Anttila S, et al: CYP1A1 and GSTM1 genetic polymorphisms and lung cancer risk in Caucasian non-smokers: a pooled analysis. Carcinogenesis 24: 875-882, 2003.

19. Cote ML, Chen W, Smith DW, Benhamou S, Bouchardy C, Butkiewicz D, Fong KM, Gené M, Hirvonen A, Kiyohara C, et al: Meta- and pooled analysis of GSTP1 polymorphism and lung cancer: a HuGE-GSEC review. Am J Epidem 169: 802-814, 2009.

20. Carlsten C, Saggo GS, Frodsham AJ, Burke W and Higgins JP: Glutathione S-transferase M1 (GSTM1) polymorphisms and lung cancer: a literature-based systematic HuGE review and metaanalysis. Am J Epidem 167: 759-774, 2008.

21. Butkiewicz D, Grzybowska E, Phillips DH, Hemminki K and Chorazy M: Polymorphisms of the GSTP1 and GSTM1 genes and PAH-DNA adducts in human mononuclear white blood cells. Environ Mol Mutagen 35: 99-105, 2000.

22. Chen Z, Li Z, Niu X, Yu Y, Lu S and Chen Z: The effect of CYP1A1 polymorphism on the risk of lung cancer: a global meta-analysis based on 71 case-control studies. Mutagenesis 26: 437-446, 2011.

23. Dialyna IA, Miyakis S, Georgatou N and Spandidos DA: Genetic polymorphisms of CYP1A1, GSTM1 and GSTT1 and lung cancer risk. Oncol Rep 10: 1829-1835, 2003. 
24. Risch A, Wikman H, Thiel S, Schmezer P, Edler L, Drings P, Dienemann H, Kayser K, Schulz V, Spiegelhalder B and Bartsch H: Glutathione S-transferase M1, M3, T1 and P1 polymorphisms and susceptibility to non-small cell lung cancer subtypes and hamartomas. Pharmacogenetics 11: 757-764, 2001

25. Lu C, Spitz MR, Zhao H, Dong Q, Truong M, Chang JY, Blumenschein GR Jr, Hong WK and Wu X: Association between glutathione $\mathrm{S}$-transferase pi polymorphisms and survival in patients with advanced non-small cell lung carcinoma. Cancer 106: 441-447, 2006.

26. Sreeja L, Syamala V, Hariharan S, Syamala VS, Raveendran PB, Sivanandan CD, Madhavan J and Ankathil R: Glutathione S-transferase M1, T1 and P1 polymorphisms: susceptibility and outcome in lung cancer patients. J Exp Ther Oncol 7: 73-85, 2008.
27. Sweeney C, Nazar-Stewart V, Stapleton PL, Eaton DL and Vaughan TL: Glutathione S-transferase M1, T1 and P1 polymorphisms and survival among lung cancer patients. Cancer Epid Biom Prev 12: 527-533, 2003.

28. Goto I, Yoneda S, Yamamoto M and Kawajiri K: Prognostic significance of germ-line polymorphisms of the CYP1A1 and glutathione S-transferase genes in patients with non-small cell lung cancer. Cancer Res 56: 3725-3730, 1996.

29. Ada AO, Kunak CS, Hancer F, Soydas E, Alpar S, Gulhan M and Iscan M: CYP and GST polymorphisms and survival in advanced non-small cell lung cancer patients. Neoplasma 57: 512-521, 2010. 